\title{
Celebrating synthesis
}

\author{
Chemical synthesis plays a critical and creative role in chemical biology, providing new chemical \\ materials to investigate biological systems.
}

The International Year of Chemistry 2011 is an initiative of the International Union of Pure and Applied Chemistry (IUPAC) and the United Nations Educational, Scientific, and Cultural Organization (UNESCO) intended to "increase the public appreciation of chemistry in meeting world needs, to encourage interest in chemistry among young people, and to generate enthusiasm for the creative future of chemistry" (http:// www.chemistry2011.org/). Organic synthesis in particular has served as a showcase for the creativity of chemists, with different routes to a natural product or unusual functional group transformations reflecting both the state of the art and a particular chemist's definition of elegance. This mindset and track record in providing tailor-made molecules have made synthetic chemistry an essential part of chemical biology.

Chemistry has played a central role in chemical biology from the start, both in outlook and in practice (Nat. Chem. Biol. 1, 3, 2005; Nat. Chem. Biol. 2, 223, 2006). The application of chemical principles and the search for mechanistic insights have largely defined the field, allowing intriguing observations to be explored in a quantitative way. The rigorous chemical characterization of small molecules and increasingly larger biomolecules has similarly allowed chemical biologists to define biological function with unprecedented molecular and spatial certainty. Although advances in all disciplines of chemistry have contributed to these endeavors, many chemical biology experiments critically rely on the use of a selective labeling strategy, the application of a small molecule as a substrate analog or synthetic inhibitor or the development of model systems to explain observations made at the cellular level. All of these depend heavily on the thoughtful design and precise construction of new molecular architectures, skills in which synthetic chemists excel.

Biomolecular labeling has advanced extensively from early nonselective reagents such as maleimides to the current wealth of protocols offering site selectivity, temporal regulation and cellular compatibility (see, for example, Nat. Chem. Biol. 5, 341, 2009). Synthetic chemistry has driven these advances, as the development of these new methods requires an understanding of functional group reactivity and of the chemical logic behind different reaction sequences as well as the synthetic capability to generate appropriate reagents and characterize the labeling chemistry, often in the complex environment of the cell. To make sense of the growing catalog of these tagging methods, a Review in this issue by Stephanopoulos and Francis provides a 'bioconjugation flowchart' that-through the simple consideration of functional groups and biological context-guides users to strategies best matched to the desired application (p. 876). By describing ways in which proteins can be modified according to established protocols, the flowchart also highlights lacunae in the bioconjugation toolbox where synthetic chemists can apply their skills in the discovery and deployment of new reactions.

A primary aim of applying defined chemical tools in chemical biology research is to achieve a greater understanding of biological systems at the molecular level. Beyond developing labeling reagents, synthetic chemists facilitate this effort by providing access to natural products, lipids or sugars, enabling exploration of their biological function. Often, modulating small pieces of the molecule-even single atoms-can test specific hypotheses about molecular interactions while simultaneously advancing chemical methodologies (see, for example, Nat. Chem. Biol. 7, 591, 2011; Nat. Chem. Biol. 4, 418, 2008 and Nat. Chem. Biol. 3, 559-564 2007). In some cases, such as in biomimetic strategies, the synthesis of a molecule in and of itself can provide insight into an outstanding biological question (Nat. Chem. Biol. 4, $535,2008)$. In addition, as described in a Perspective in this issue by Razzak and DeBrabander, biomimetic syntheses can change our understanding of synthetic rules and encourage the search for new natural products that 'should' exist on the basis of known chemical principles (p. 865).

One of the foundational ideas of chemical biology was "to identify a small molecule partner for every gene product" to unravel the secrets of biological networks and function (Bioorg. Med. Chem. 6, $1127,1998)$. For many scientists, chemical biology has become synonymous with the application of small molecules as tools or probes to interrogate biology. Public and private small-molecule libraries have been constructed to facilitate small-molecule discovery and are widely used by chemists and biologists alike. However, we remain far from the original goal of identifying one molecule for every protein, in large part because we lack the requisite molecular diversity. This shortfall points to an urgent need for creative, efficient and highyielding methods to access new molecules, particularly those that diverge from existing scaffolds. Surprisingly, according to feedback from both formal interactions with referees of Nature Chemical Biology papers and informal conversations at conferences, synthetic efforts toward this end are generally not viewed as within the scope of a chemical biology journal. From our perspective, contributions that explore chemical space-whether built on DNA scaffolds (Nat. Chem. Biol. 5, 647, 2009) or performed in a round-bottom flask (Nat. Chem. Biol. 6, 199, 2010)-are not only relevant but also vital to chemical biology research and certainly appropriate for the journal's readership.

It may seem unnecessary to discuss the importance of chemistry with members of a community initiated in large part by chemists (Nat. Chem. Biol. 6, 847, 2010). Yet too often, synthetic protocols are passed over in presentations or relegated to supplementary information in published papers. Appropriate validation of standards and probes can seem to be of secondary importance, and small-molecule libraries are viewed as simply commercial products rather than as living resources being actively shaped by chemists around the globe. The International Year of Chemistry offers a useful reminder for chemical biologists to reflect on the unique strengths and contributions of chemistry rather than viewing synthetic chemistry as a routine process that happens only as a backdrop for scientific research. By identifying new opportunities for synthetic solutions, chemical biologists can both take pride in their chemical heritage and take responsibility for understanding and creatively manipulating the chemical matter they employ. 